\title{
An expanded palette of fluorogenic HaloTag probes with enhanced contrast for targeted cellular imaging
}

\author{
Sylvestre P.J.T. Bachollet, ${ }^{\mathrm{a}}$ Yuriy Shpinov, ${ }^{\mathrm{a}}$ Fanny Broch, ${ }^{\mathrm{a}}$ Hela Benaissa, ${ }^{\mathrm{a}}$ Arnaud Gautier,,${ }^{\mathrm{a}, \mathrm{b}}$ Jean-Maurice Mallet ${ }^{\mathrm{a}}$ \\ and Blaise Dumat ${ }^{\mathrm{a} *}$ \\ a Laboratoire des biomolécules, LBM, Département de chimie, École normale supérieure, PSL University, Sorbonne Université, CNRS, 75005 \\ Paris, France. \\ ${ }^{\mathrm{b}}$ Institut Universitaire de France (IUF), 1 rue Descartes, 75005 Paris \\ *Corresponding author : $\underline{\text { blaise.dumat@ens.psl.eu }}$
}

\section{Introduction}

Fluorescence imaging has become an invaluable tool to study the structure and dynamics of living systems due to its high sensitivity (down to the single molecule), high temporal and spatial resolution and to its biocompatibility, allowing non-invasive imaging of samples in physiological conditions. Numerous fluorescent molecular probes have been developed over time and organic chemistry offers virtually endless possibilities to tailor fluorophores for each application. ${ }^{1}$ Nonetheless, since the discovery of the Green Fluorescent Protein (GFP), fluorescent proteins (FPs) have taken a prominent place in the field of bioimaging thanks to the unparalleled targeting selectivity of genetic encoding. A large variety of FPs has since been developed with emission spanning the whole visible spectrum and optimized optical and biochemical properties paving the way to multicolor and complex imaging experiments. ${ }^{2}$ More recently, hybrid chemogenetic systems have appeared, that associate a genetically encoded self-labeling protein tag with a small molecular fluorophore linked either covalently or non-covalently to the protein. ${ }^{3-7}$ Such combination benefits from the genetic targeting of FPs and from the structural diversity and versatility of organic chromophores and it offers better spatial and temporal control over the fluorescent labeling compared to fluorescent proteins that are continuously expressed and fluorescent. It may nonetheless suffer from common problems associated with molecular probes, including the risk of off-target binding, yielding non-specific signal, and the need to wash the excess of dye. To alleviate these issues, advanced chemogenetic reporters are built on fluorogenic probes that only become fluorescent when bound to the cognate protein tags. ${ }^{8-11}$ Considerable efforts have thus been put in the development of fluorogens with diverse optical properties and on their association with protein tags, in particular the most commonly used SNAP-tag ${ }^{12-15}$ and HaloTag. ${ }^{16-21}$ The latter is, to date, one the most useful tag for covalent protein labeling with high selectivity and fast reaction kinetics. Its chemically simple and lipophilic chloroalkane ligand also facilitates the synthesis of the dedicated probes as well as their cell permeability. ${ }^{22}$

The HaloTag is derived from a bacterial alkane dehalogenase and was built and optimized (up to the last iteration HaloTag7) to bind xanthene dyes and especially rhodamine derivatives with high reaction rates $\left(\mathrm{k}_{2}=1.9 \times 10^{7} \mathrm{M}^{-}\right.$ ${ }^{1} \mathrm{~S}^{-1}$ for TMR-Halo at $\left.25^{\circ} \mathrm{C}\right){ }^{3,23}$ This has driven a lot of the research on HaloTag probes towards rhodamines and, more particularly, fluorogenic Si-Rhodamines taking advantage of the polarity-sensitive spirocyclization reaction. ${ }^{19,20,22,24-26}$ This approach certainly yields highly efficient fluorescent chemogenetic reporters but has narrowed their chemical diversity. Alternative strategies to develop fluorogenic HaloTag probes have relied on the intrinsic viscosity and/or polarity-sensitive emission of dipolar fluorophores. ${ }^{16,17,27}$ The approach was pioneered by Kool and coworkers who developed a dimethylamino-styrylpyridinium dye with a viscositysensitive emission that was activated upon reaction with HaloTag thanks to the immobilization inside the protein binding pocket. This so-called "molecular rotor" design allows exploring a much wider chemical space: the fluorescence activation mechanism is applicable to a large variety of dipolar structures with electron-donating and withdrawing groups connected through a flexible conjugated bond and is commonly used to develop biomolecular probes. ${ }^{28-33}$ Based on this strategy, we have previously designed a series of red and far-red emitting fluorogenic HaloTag probes (Figure 1A). ${ }^{21}$ After reaction with HaloTag, the probes are covalently tethered to the protein and exhibit large enhancements of their fluorescence emissions compared to their free form in water 
(up to 156-fold). The two most promising fluorogens Red-Halo2 and NIR-Halo1 enabled wash-free imaging of the nucleus and actin in live cells by targeting HaloTag-NLS and HaloTag-LifeAct fusion proteins respectively. We nonetheless observed a low nonspecific signal in the cytoplasm that may prove detrimental when imaging lowabundant or cytosolic proteins.

Herein, taking advantage of the versatile design, we report an expanded palette of HaloTag fluorogens designed to cover a wider emission range from green to red and to minimize the non-specific signal. The new set of probes was characterized in vitro by absorption and fluorescence spectroscopies and in cell imaging by confocal microscopies. Some new analyses were also performed on the previously reported Red-Halo2 and NIR-Halo1 probes for comparison purposes. The photophysical properties were studied in vitro in glycerol and in presence of HaloTag to confirm their viscosity-dependent emission and fluorogenic reaction with the protein tag. The selectivity of the probes in wash-free imaging experiments was then studied in cells and, taking advantage of the superior contrast of the newly designed probes, we applied them to image of a variety of cellular organelles and proteins.

\section{Results and discussion}

Design and synthesis. The previously reported Red-Halo and NIR-Halo probes cover an emissive range from red to far-red. In order to cover a lower wavelength range, we have modified Red-Halo2 to introduce two lesser deficient electron-withdrawing groups, 2-cyanoacetic acid or imidazolinone, to form, respectively, two green and yellow emitting derivatives CCVJ-Halo and Y-Halo. 2-Cyanoacetic acid forms the CCVJ fluorophore which is a classical molecular rotor with a green emission used as a fluorogenic protein probe and it presents a readily available carboxylic acid function for conjugation with the HaloTag ligand. ${ }^{13,34,35}$ CCVJ was already used with HaloTag but with a different ligand unit which does not readily make the probe fluorogenic upon reaction with the protein and was instead used to detect protein aggregation. ${ }^{27}$ Imidazolinone on the other hand is the electron withdrawing group found in the GFP chromophore and, in aminated derivatives, it was reported to afford yellowemitting compounds. ${ }^{36}$

A

Previous work
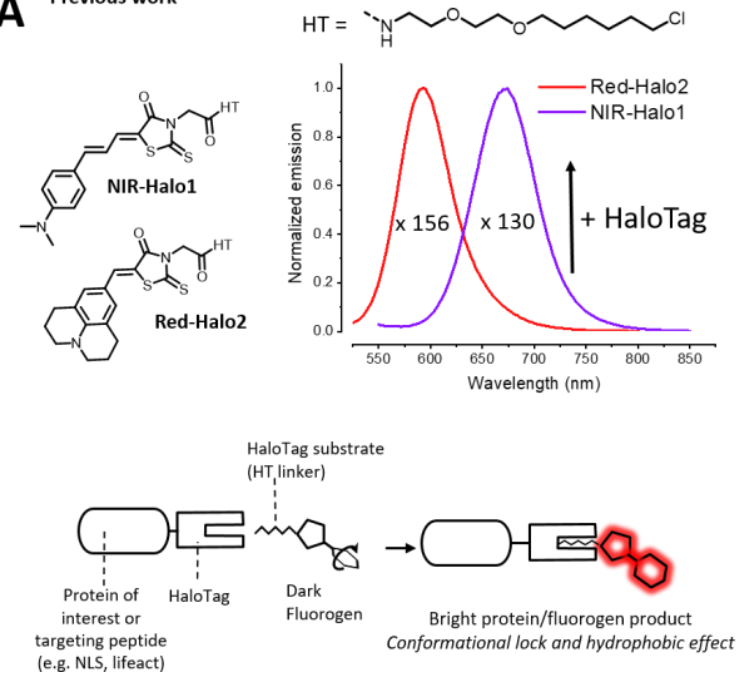

B Structural modification to tune the optical properties and improve the imaging contrast
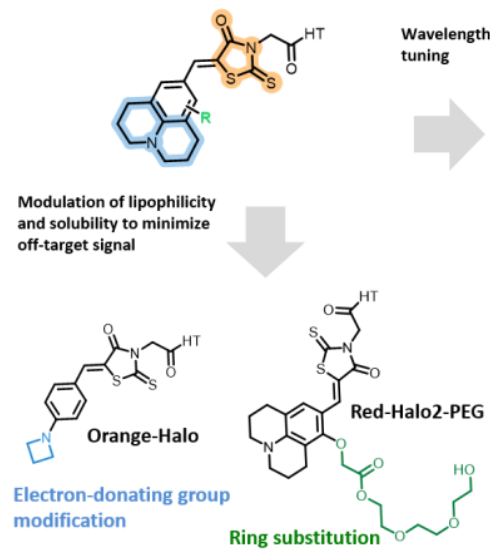
Wavelength modification

Ring substitution

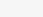
Electron-withdrawing group modification<smiles>[Y][R]C(=O)CN1C(=O)SC(=CC)C1=O</smiles>
Red-Halo2

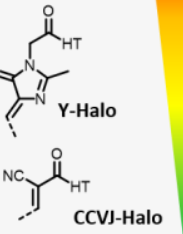
CCVJ-Halo

Figure 1. Design and structures of the fluorogenic HaloTag probes.

(A) structures of two of the previously reported probes with their fluorescence emission spectra and fluorescence enhancement upon reaction with HaloTag and general schematic description of the fluorogenic reaction with HaloTag. (B) Structural modifications and structures of the new probes.

In order to minimize the unspecific signal observed with Red-Halo2 and NIR-Halo1, we have set out to develop analogs with similar optical properties as Red-Halo2 but with lower lipophilicity. Factors governing the nonspecific binding of a dye to intracellular components are difficult to predict and depend in part on the net charge (e.g. positively charged rhodamine binding to mitochondria) and on the lipophilic/hydrophilic balance. The Halo 
dyes being neutral structures with poor water solubility, we hypothesized that their lipophilic character is one of the driving force of the non-specific binding by leading the probes to accumulate into lipid structures such as the endoplasmic reticulum (ER) membrane. As a first strategy, we have synthesized Red-Halo2-PEG with a similar core structure than Red-Halo2 but bearing a solubilizing triethylene glycol chain on the aniline ring. In a second approach, we have introduced an azetidine donating amino group instead of the julolidine (Figure 1). Azetidine was previously reported as an efficient locked amino donating group since the 4-membered cycle strain prevents the formation of a "twisted intramolecular charge transfer" (TICT) state and it may advantageously replace julolidine while being slightly less lipophilic. ${ }^{20}$ Azetidine is also less electron-donating than julolidine and yielded the blue-shifted orange-emitting Orange-Halo. Using inexpensive building blocks and reagents, the probes can be obtained in straightforward 2 to 4 steps synthetic procedures detailed in the supplementary information (Scheme S1-S4).

Photophysical characterization in glycerol. All probes display typical molecular rotor features, with a donor and acceptor group connected through a flexible bond, and we have previously evidenced the fluorogenic behavior of Red-Halo2 and NIR-Halo1 in presence of proteins while CCVJ is an archetypical rotor probe. We have nonetheless confirmed the molecular rotor properties (i.e. the viscosity-dependent emission) for the whole series of probes by measuring their spectral properties in water and in glycerol (Figure 2 and Table 1).

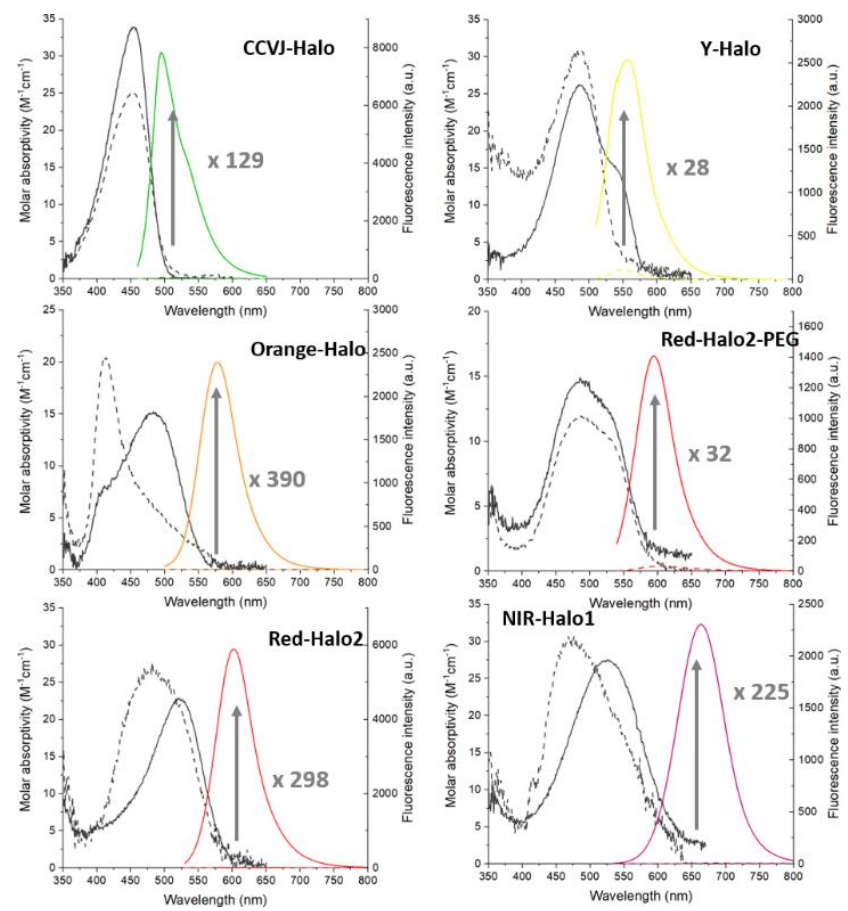

Figure 2. Viscosity-dependent emission of the molecular rotor probes.

Absorption (black lines) and fluorescence emission (colored lines) in water (dashed lines) and in glycerol (full lines) of the Halo probes. On each panel is also indicated the fluorescence enhancement factor $F / F_{0}$ where $F$ is the fluorescence intensity in glycerol and $F_{0}$ the fluorescence in water.

As expected from the rational design, all probes are weakly fluorescent in water but their emission is strongly enhanced in glycerol with quantum yields ranging from 9 to $20 \%$, in accordance with an increase in viscosity. The highest brightness in glycerol is observed for CCVJ-Halo with a 129-fold enhancement and a green emission centered on $495 \mathrm{~nm}$, which confirms that CCVJ conjugated to the HaloTag ligand retains its molecular rotor properties. Y-Halo exhibits a slightly higher residual fluorescence in water as well as a lower brightness in glycerol which results in a lower flurogenicity. Although the azetidine is slightly less electron-donating than the julolidine, Orange-Halo is expected to have spectral properties close to those of Red-Halo2 but its absorption in water is unexpectedly blue-shifted (409 $\mathrm{nm}$ ). In glycerol however, a large bathochromic shift is observed resulting in an absorption spectrum more akin to the parent Red-Halo2 structure with a corresponding emission centered on 
$578 \mathrm{~nm}$, blue-shifted by $24 \mathrm{~nm}$ compared to Red-Halo2. The activation of the fluorescence in glycerol combined with the shift of the excitation maximum result in the highest enhancement of the series (390-fold), although the brightness remains lower than that of CCVJ-Halo. Red-Halo2 and NIR-Halo1 exhibit similar behaviors as were observed in presence of HaloTag with a red-shift of the absorption between water and glycerol and a large fluorescent enhancement of their red and far-red emission centered on 602 and $664 \mathrm{~nm}$ respectively. ${ }^{21}$ The spectral properties of Red-Halo2-PEG are essentially conserved compared to its parent compound but its brightness is significantly lower resulting in a lower fluorogenicity.

Table 1. Photophysical properties in Glycerol

maximum absorption wavelength $\lambda_{a b s}$; molar absorptivity $\varepsilon$; maximum emission wavelength $\lambda_{e m}$; fluorescence quantum yield $\Phi_{F}$; emission brightness $\varepsilon \cdot \Phi_{F}$; fluorescence enhancement between water and glycerol $F / F_{0}$ where $F$ is the fluorescence in glycerol and $F_{0}$ the fluorescence in water measured at a $2 \mu \mathrm{M}$ concentration in dye.

\begin{tabular}{c|cccccc}
\hline & $\begin{array}{c}\boldsymbol{\lambda}_{\text {abs }} \\
(\mathbf{n m})\end{array}$ & $\begin{array}{c}\boldsymbol{\varepsilon} \\
\left(\mathbf{M}^{-1} \mathbf{c m}^{-1}\right)\end{array}$ & $\begin{array}{c}\boldsymbol{\lambda}_{\mathrm{em}} \\
(\mathbf{n m})\end{array}$ & $\boldsymbol{\Phi}_{\mathrm{F}}$ & $\boldsymbol{\varepsilon} \cdot \boldsymbol{\Phi}_{\mathrm{F}}$ & $\mathbf{F} / \mathbf{F}_{\mathbf{0}}$ \\
\hline CCVJ-Halo & 455 & 33900 & 495 & 0.19 & 6441 & 129 \\
Y-Halo & 487 & 26200 & 556 & 0.099 & 2594 & 28 \\
Orange-Halo & 486 & 15200 & 578 & 0.14 & 2128 & 390 \\
Red-Halo2-PEG & 520 & 14900 & 595 & 0.086 & 1281 & 32 \\
Red-Halo2 & 521 & 22900 & 602 & 0.20 & 4580 & 298 \\
NIR-Halo1 & 527 & 27500 & 664 & 0.091 & 2503 & 225 \\
\hline
\end{tabular}

Reaction with HaloTag and photophysical properties. The properties in glycerol confirm the potential of the probes to be used as fluorogenic biomolecular probes thanks to a viscosity-sensitive emission and we next studied the reaction of the probes with a GST-HaloTag (GST-HT) protein in vitro and the resulting fluorescence properties of the formed complexes. The results are summarized in table 2 and Figure 3.

Table 2. Photophysical properties of the dyes bound to GST-HaloTag.

$\lambda_{a b s}$ and $\lambda_{e m}$ : maximum absorption and emission wavelength, $\varepsilon$ : molar absorptivity coefficient, FWHM: Full width at Half maximum, $\Phi_{F}$ : fluorescence quantum yield, $\varepsilon \cdot \Phi_{F}$ : brightness, $k_{2}$ : second order reaction rate constant, $F / F_{0}$ : Fluorescence enhancement factor where $F$ and $F_{0}$ are the integrated fluorescence intensities in presence of HaloTag and in water respectively.

\begin{tabular}{|c|c|c|c|c|c|c|c|c|}
\hline & $\begin{array}{c}\lambda_{\text {abs }} \\
(\mathrm{nm})\end{array}$ & $\begin{array}{c}\varepsilon \\
\left(10^{3} \mathrm{M}^{-1} \mathrm{~cm}^{-1}\right)\end{array}$ & $\begin{array}{c}\lambda_{\mathrm{em}} \\
(\mathrm{nm})\end{array}$ & $\begin{array}{l}\text { FWHM } \\
(\mathrm{nm})\end{array}$ & $\Phi_{\mathrm{F}}$ & $\varepsilon \cdot \Phi_{\mathrm{F}}$ & $\begin{array}{c}\mathbf{k}_{2}{ }^{\mathrm{b}} \\
\left(10^{3} \mathrm{M}^{-1} \mathrm{~s}^{-1}\right)\end{array}$ & $F / F_{0}$ \\
\hline CCVJ-Halo & 458 & 31.8 & 498 & 54 & 0.014 & 445 & $1.0 \pm 0.1$ & 15 \\
\hline Y-Halo & 489 & 34.9 & 562 & 50 & 0.015 & 524 & n.d. & 12 \\
\hline Orange-Halo & 487 & 16.2 & 574 & 64 & 0.02 & 324 & $0.45 \pm 0.03$ & 48 \\
\hline Red-Halo2-PEG & 504 & 14.5 & 588 & 65 & 0.05 & 725 & $0.29 \pm 0.04$ & 32 \\
\hline Red-Halo2 ${ }^{\mathrm{a}}$ & 515 & 21.0 & 592 & 60 & 0.17 & 3600 & $1.3 \pm 0.2$ & 156 \\
\hline NIR-Halo1 $^{\text {a }}$ & 526 & 25.8 & 671 & 68 & 0.053 & 1400 & $1.7 \pm 0.04$ & 130 \\
\hline
\end{tabular}

Measurement conditions: $1 \mu \mathrm{M}$ dye with $1.7 \mu \mathrm{M}$ GST-HT in pH 7.4 phosphate buffer containing $10 \mathrm{mM}$ Phosphate and $100 \mathrm{mM}$ NaCl. ${ }^{a}$ Data taken from ref. ${ }^{21}$ except for $\mathrm{k}_{2}$ values. ${ }^{\mathrm{b}}$ Average of duplicate experiments.

All probes undergo a fluorogenic reaction reaction with GST-HT, which allowed us to measure the reaction rate constants by following the increase of fluorescence over time (Figure $3 A \& B$ ). In presence of a large excess of protein, the reactions obey a classical pseudo-first-order exponential law with the exception of Y-Halo and the calculated second order reaction rate constants are comprised between $0.2910^{3}$ and $1.710^{3} \mathrm{M}^{-1} \mathrm{~s}^{-1}$. The slowest reaction rates were observed for Orange-Halo and Red-Halo2-PEG which can be explained in part by solubility and steric hindrance issues. The unexpectedly blue-shifted absorption of Orange-Halo suggests the formation of aggregates which may also explain the slow reaction kinetics and Red-Halo2-PEG bears a PEG chain that may hinder the reaction. On the other hand, the fastest reaction rate is observed for NIR-Halo1 in which the extended $\pi$-system takes the bulky aromatic group further from the reaction site. The reaction with Y-Halo is almost instantaneous with an immediate sharp increase of fluorescence that is followed by a slower decrease of intensity that stabilizes after a few minutes (Figure 3B). It is possible that Y-Halo undergoes a first non-covalent association with the protein yielding a highly fluorescent complex followed by the covalent enzymatic reaction that leads to a compound with lower fluorescence. To assume a pseudo-first order constant, the kinetics experiments were carried-out at $100 \mathrm{nM}$ in Halo probes with a 20-fold excess of protein. In more standard 
experimental conditions ( $1 \mu \mathrm{M}$ probe and 1.7 eq of protein) the evolution of the fluorescence over time confirms the very fast reaction of Y-Halo but with stable fluorescence intensity (Figure S1). Apart from Y-Halo, the reaction rates of the probes are slower than that of rhodamine-based HaloTag ligands but they are in the same range or faster than that observed for other reported solvatochromic HaloTag probes ${ }^{16,18}$ and for alternative self-labeling protein tags such as SNAP-tag, CLIP-tag and PYP-tag. ${ }^{4,5,10,12}$
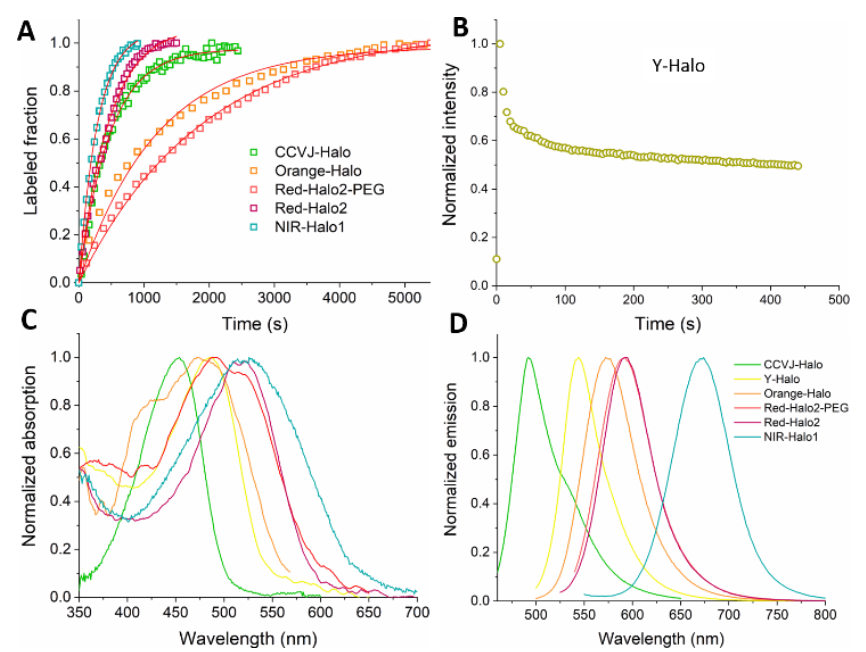

Figure 3. Reaction of the Halo probes with GST-HaloTag.

$(\mathrm{A}, \mathrm{B})$ Reaction kinetics of the Halo probes with GST-HT. [dye] $=100 \mathrm{nM},[\mathrm{GST}-\mathrm{HT}]=2 \mu \mathrm{M}, \mathrm{T}=298 \mathrm{~K}$. Data was fitted to an exponential rise function (red lines) with the exception of Y-Halo. Normalized absorption (C) and emission (D) of the Halo probes bound to HaloTag. [Dye] = $1 \mu \mathrm{M}[\mathrm{GST}-\mathrm{HT}]=1.7 \mu \mathrm{M}$.

After reaction with HaloTag, the spectral properties of the bound probes are essentially similar to those in glycerol but with lower fluorogenicities (Table 2). CCVJ-Halo and Y-Halo display comparable 12 to 15 -fold fluorescence activation and while Red-Halo2-PEG and Orange-Halo perform slightly better, the four new probes have lower fluorescence enhancement factors than their parent compound Red-Halo2 (156-fold), which stems essentially from lower fluorescence quantum yields. CCVJ-Halo, Y-Halo and Orange-Halo display absorption spectra centered on $458 \mathrm{~nm}, 489 \mathrm{~nm}$ and $487 \mathrm{~nm}$ respectively, which allows for an optimal excitation with the 458 and $488 \mathrm{~nm}$ Argon laser lines commonly available on microscopes. The Red-Halo2 derivatives and NIR-Halo1 can be efficiently excited with the 488 or $514 \mathrm{~nm}$ laser lines. Due to their flexible structures that increase the number of available vibrational energy levels, the Halo dyes inherently display broad emission peaks. The more flexible structure NIR-Halo1 displays the broadest peak, with a FWHM of $68 \mathrm{~nm}$. The emission peaks of RedHalo2, Red-Halo2-PEG and Orange-Halo have comparable, albeit slightly narrower, breadth (Figure 3C). Although considerable progress has been made in the analysis and spectral deconvolution of multicolor images, these large emission ranges may render their discrimination from other fluorophores more difficult in multiplexed experiments. On the other hand, the green and yellow derivatives, CCVJ-Halo and Y-Halo, exhibit narrower emission peaks with FWHM of 54 and $50 \mathrm{~nm}$ that could make them more easily applicable in multicolor imaging. The new HaloTag fluorogens display promising properties with fluorogenic emissions of varied colors and adequate spectral properties for microscopy. The performance of the probes was next assessed in wash-free live cell imaging with a particular emphasis on the imaging contrast.

Cellular imaging. The probes were first applied to the cellular imaging of live Hela cells transfected with HaloNLS. This strategy was successfully used in our previous report and the staining of a well-defined region of the cells allows comparing the performance of the various probes by measuring the contrast between the specific nuclear signal and a possible non-specific signal in the cytoplasm. 

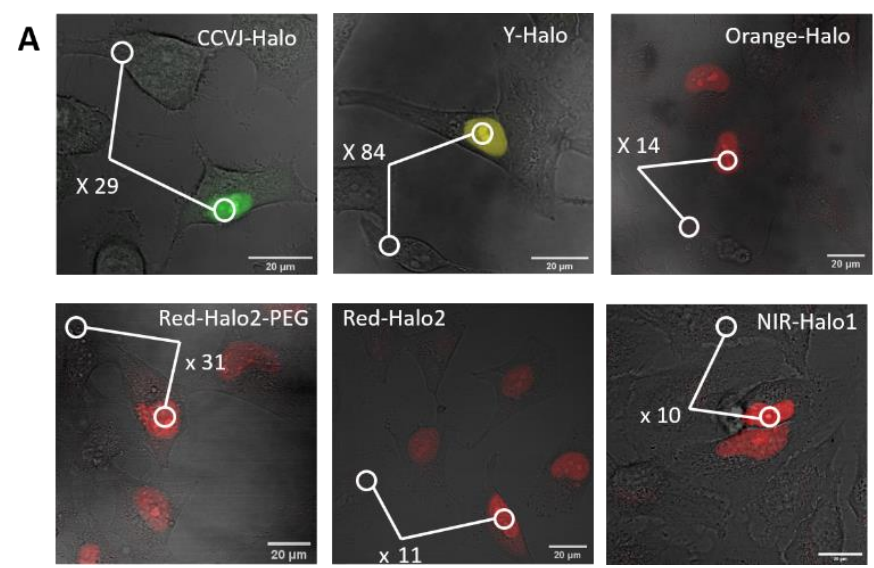

B

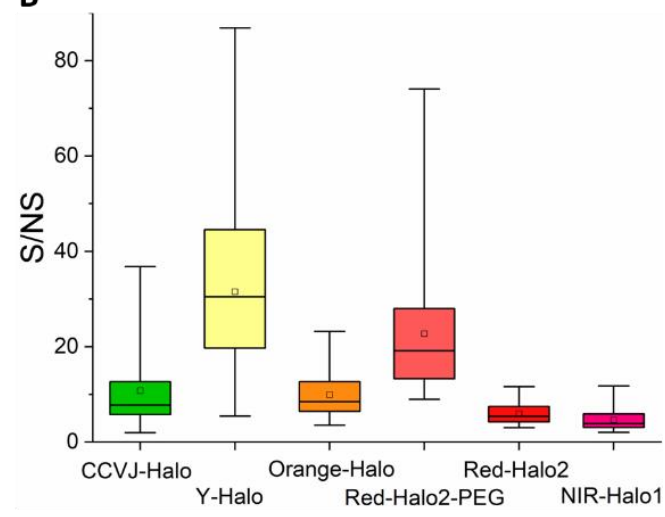

Figure 4. Selectivity and contrast of the Halo probes in cellular imaging.

(A) Live confocal imaging of Hela cells transfected with Halo-NLS plasmid and incubated with $0.5 \mu \mathrm{M}$ of fluorogenic Halo probe. On each panel is displayed the contrast ratio between the intensity in the nucleus and the non-specific signal measured in the cytoplasm of a nontransfected cell on the same image. $\lambda_{\text {exc }}=514 \mathrm{~nm}$ except for CCVJ-Halo (458 nm) and Y-Halo (488 nm). Scale bar : $20 \mu \mathrm{m}$ (B) Contrast ratio of the specific ( $\mathrm{S}=$ average intensity in the nucleus) over the non-specific signal (NS = averaged intensity in a cytosolic region of a nontransfected cell). Box and whiskers plot with center lines and squares showing the median and average values for $n$ cells respectively. Box delimits the $25^{\text {th }}$ and $75^{\text {th }}$ percentiles and the whiskers show the max and min values. CCVJ-Halo: $n=64$ from four experiments, Y-Halo: $n=$ 43 from two experiments, Red-Halo2-PEG: $n=94$ from four experiments, Orange-Halo: $n=91$ from three experiments, Red-Halo2: $n=54$ from three experiments, NIR-Halo1: $n=72$ from four experiments.

The imaging was performed using a similar protocol as for Red-Halo2: after transfection the cells were stained with $0.5 \mu \mathrm{M}$ of Halo probe and were imaged after 30 to 60 minutes without changing the medium or washing the excess dye. After incubation, the cells showed a good viability with no sign of cytotoxicity and all four new probes were successful in selectively staining the nucleus (Figure 3A). To assess the performance of our dye in wash-free imaging protocols, we have compared the specific signal $(\mathrm{S})$ in the nuclei with the non-specific signal (NS) in the cytoplasm of an untransfected cell. Untransfected cells were chosen as reference since transfected cells may exhibit some fluorescence in the cytoplasm due to the reaction of the fluorogen with newly synthesized proteins in the cytoplasm which is not per se an off-target binding. The contrast ratio (S/NS) is given on each panel of figure 3A. For a more precise analysis of the probes selectivity, we have calculated the contrast on a larger set of cells and the distribution of the results is given as a box plot on figure 3B. The specific signal (and thus the related contrast) is highly dependent on the transfection level, which causes a large dispersion of the values. Nonetheless, by looking at the average value or at the highest contrast to be expected with a good transfection level, these results clearly show that the newly designed probes with lower lipophilicity Red-Halo2PEG and Orange-Halo indeed improve the contrast of the parent compound Red-Halo2. Imaging of wild-type Hela cells stained with Red-Halo2, Red-Halo2-PEG and Orange-Halo confirm these observations (Figure S2). Despite the minute structural change between Red-Halo2 and Orange-Halo, the latter yields a cytoplasmic signal 3 -fold lower than the former and the effect is even more pronounced with the pegylated derivatives with a 6 fold higher cytoplasmic signal for Red-Halo2 compared to Red-Halo2-PEG, which is well in line with the relative distribution of the S/NS ratios. Interestingly and although they were not specifically designed for this, the CCVJHalo and Y-Halo derivatives also exhibits improved S/NS ratios that reach remarkably high values for the latter. These results stress the difficulty of designing efficient fluorogenic probes and the necessity to balance in vitro and cellular properties: a high fluorescence enhancement factor in vitro is an important figure of merit for a fluorogenic probe, yet it does not always translate into a high contrast in cellular imaging due to the possibility of off-target fluorogen activation. Here, despite its superior optical properties measured in cuvette, Red-Halo2 is also the most prone to off-target activation in cells yielding a higher non-specific signal. On the other hand, the new probes exhibit excellent contrast values that are not directly correlated to the relative brightness or fluorescence enhancement values. 
In order to take advantage of the increased contrast, we have finally used the palette of HaloTag probes to image of variety of cellular components in addition to the HaloTag-NLS nuclear labeling discussed above (Figure 5 and Figure S3).
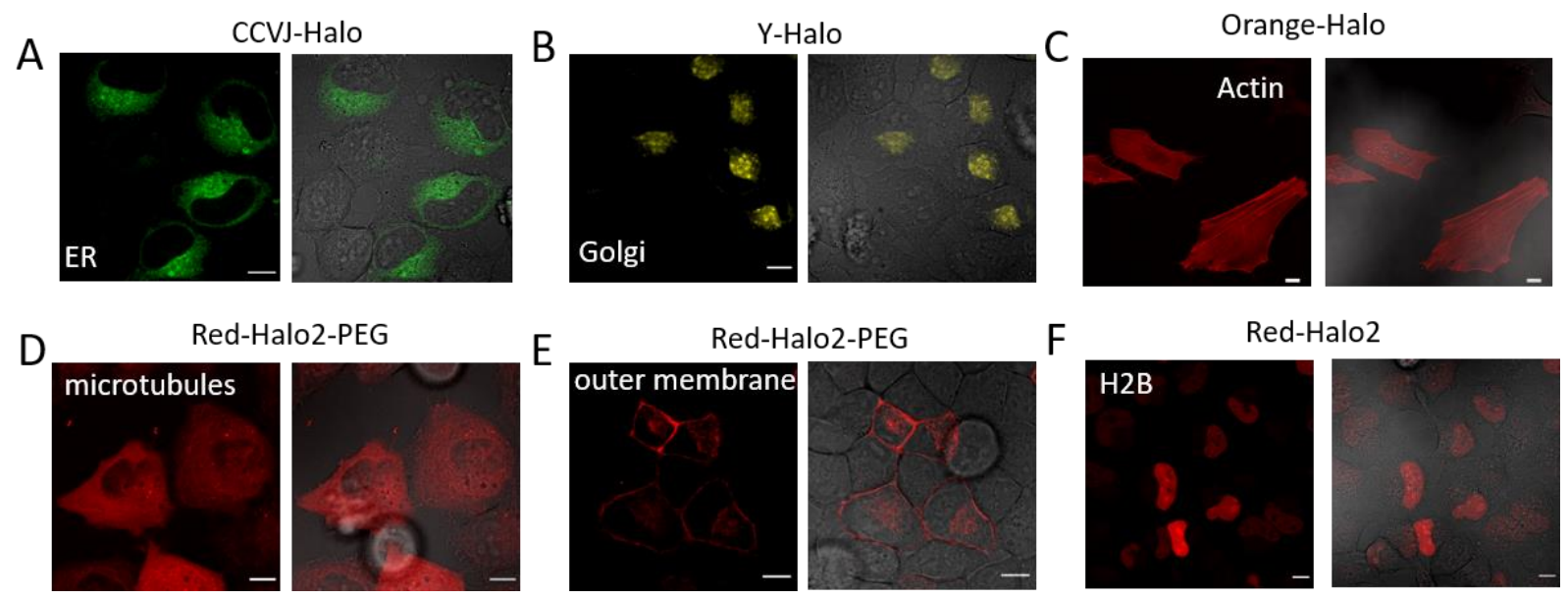

Figure 5. Targeted organelles and proteins imaging.

Live wash-free confocal imaging of Hela cells transfected with either H2B-HaloTag (nucleus), MAP4-HaloTag (microtubules), PGDFR-HaloTag (external membrane), ER-HaloTag (endoplasmic reticulum), HaloTag-Lifeact (actin) or Golgi-HaloTag (Golgi apparatus) and incubated with $0.5 \mu \mathrm{M}$ of CCVJ-Halo (A), Y-Halo (B) or Orange-Halo (C) Red-Halo2-PEG (D,E) and Red-Halo2 (F). Each panel diplays the fluorescence image (left) and the merged fluorescence and brightfield images (right). Non-transfected cells are visible on the merged images to evidence the selective labeling. $\lambda_{\text {exc }}=514 \mathrm{~nm}$ except for CCVJ-Halo $(458 \mathrm{~nm})$ and Y-Halo $(488 \mathrm{~nm})$ Scale bar: $10 \mu \mathrm{m}$.

We have tested the imaging of the ER, the golgi apparatus, histone $\mathrm{H} 2 \mathrm{~B}$ for a different nuclear staining strategy, microtubules, the outer membrane and actin. In agreement with the results obtained with Halo-NLS, the probes afforded images with good contrast despite the wash-free protocols. The parent compound Red-Halo2 still proved efficient to image the nucleus or the cytoskeleton using H2B-HaloTag or MAP4-HaloTag, affording contrasted and detailed images thanks to its brightness (Figure $4 \mathrm{~F}$ and Figure S3). In addition to opening additional possibilities in terms of emission range, the new probes, thanks to their improved selectivity, enabled the imaging of cytoplasmic organelles (Figure 5A\&B), which would have been rendered difficult with the original Red-Halo2.

\section{Conclusion}

The fluorogenic HaloTag probes reported here enrich a palette of hybrid chemogenetic reporters that, combined with the previously reported ones, covers the green to far-red emission range. The newly designed probes offer a remarkable suppression of the non-specific signal enabling no-wash imaging of a variety of intracellular targets with excellent contrast. Recent reports suggest that HaloTag may be mutated to better fit solvatochromic or molecular rotor probes ${ }^{18,37}$ Engineering of the HaloTag protein may thus be envisioned in order to improve the association with our fluorogens and to reach higher quantum yields and faster reaction kinetics.

\section{Experimental procedures}

Probes synthesis and characterization. Detailed synthetic procedures and chemical analyses are available in the supplementary information.

Photophysical properties with HaloTag. The fluorogenic dyes were dissolved in DMSO at a stock concentration of $0.5 \mathrm{mM}$. The interaction of the probes with Halotag was assessed by incubating the dye at $1 \mu \mathrm{M}(0.4 \mu \mathrm{L}$ of stock solution) concentration with $1.7 \mu \mathrm{M}$ (ca. $6 \mu \mathrm{L}$ of commercial stock solution) of protein in a $200 \mu \mathrm{L}$ working volume of $\mathrm{pH}$ 7.4 Phosphate buffer (10 mM phosphate with $100 \mathrm{mM} \mathrm{NaCl}$ ). Fluorescence spectra were recorded 
at regular time intervals until completion of the reaction. The different photophysical properties of the proteinbound probes were then measured.

Kinetics study of the labeling reactions. The second-order rate constants of the reactions were calculated by monitoring the fluorescence intensity during the reaction of the probes with a large excess of protein to assume a pseudo first-order kinetics law according to the following procedure.

GST-HT $(2 \mu \mathrm{M})$ was added to a $50 \mu \mathrm{L}$ solution of Halo probe $(100 \mathrm{nM})$ in $\mathrm{pH} 7.4$ Phosphate buffer (10 mM Phosphate, $100 \mathrm{mM} \mathrm{NaCl}$ ). The fluorescence intensity (excitation and emission at the maximum absorption and emission wavelengths of each probe) was then recorded over time until completion of the reaction with a $5 \mathrm{~s}$ to $20 \mathrm{~s}$ step depending on the probe. The second order rate constants were then calculated according to a previously reported model: ${ }^{7}$ the fluorescence was converted to the labeled fraction according to the following equation:

$$
\text { Labeled fraction }=\left(F-F_{0}\right) /\left(F_{\max }-F_{0}\right)
$$

Where $F_{\max }$ and $F_{0}$ are the maximum and initial fluorescence intensity respectively. Using Origin 2019 software, the data was fitted to the following equation to calculate the pseudo first-order rate constant kobs:

$$
\text { Labeled fraction }=1-\exp \left(-k_{\text {obs }} t\right)
$$

The second order rate constant $k_{2}$ was then obtained by dividing $k_{o b s}$ with the protein concentration.

Molecular Biology. Detailed construction of the plasmids used in this study are described in the supplementary information.

Confocal microscopy. Hela cells were grown overnight on 8-well polymer $\mu$ slides from Ibidi (\#1.5 polymer coverslip, tissue culture treated) at $20 \mathrm{k}$ to $50 \mathrm{k}$ cells/well in $300 \mu \mathrm{L}$ of MEM culture medium (Gibco) supplemented with $10 \% \mathrm{fcs}$, sodium pyruvate and non-essential amino acids. Cells were then transfected with the desired plasmid using Fugene 6 (Promega Corp.) according to the manufacturer's protocol (200 ng of DNA per well with a 3:1 fugene to DNA ratio). After 24 hours, the medium was changed and replaced with DMEM (no phenol red) and the cells were incubated 30-60 minutes with the dyes and imaged live on a Zeiss LSM710 laser scanning confocal microscope equipped with a Plan apochromat 40X/1.4 NA objective. Images were acquired using Zen 2009 software and then processed using ImageJ.

\section{Acknowledgements}

This work was supported by the Agence Nationale de la Recherche (ANR-18-CE44-0006) and the European Research Council (ERC-2016-CoG-724705 FLUOSWITCH).

\section{Supplementary information}

Supplementary figures, materials and methods, chemical synthesis and molecular biology procedures are available in supplementary information.

\section{References}

(1) Lavis, L. D.; Raines, R. T. Bright Ideas for Chemical Biology. ACS Chem. Biol. 2008, 3 (3), 142-155.

(2) Day, R. N.; Davidson, M. W. The Fluorescent Protein Palette: Tools for Cellular Imaging. Chem. Soc. Rev. 2009, 38 (10), 2887-2921.

(3) Los, G. V.; Encell, L. P.; McDougall, M. G.; Hartzell, D. D.; Karassina, N.; Zimprich, C.; Wood, M. G.; Learish, R.; Ohana, R. F.; Urh, M.; et al. HaloTag: A Novel Protein Labeling Technology for Cell Imaging and Protein Analysis. ACS Chem. Biol. 2008, 3 (6), 373-382.

(4) Keppler, A.; Gendreizig, S.; Gronemeyer, T.; Pick, H.; Vogel, H.; Johnsson, K. A General Method for the Covalent Labeling of Fusion Proteins with Small Molecules in Vivo. Nat. Biotechnol. 2003, 21 (1), 86-89. 
(5) Gautier, A.; Juillerat, A.; Heinis, C.; Corrêa, I. R.; Kindermann, M.; Beaufils, F.; Johnsson, K. An Engineered Protein Tag for Multiprotein Labeling in Living Cells. Chem. Biol. 2008, 15 (2), 128-136.

(6) Hoelzel, C. A.; Zhang, X. Visualizing and Manipulating Biological Processes by Using HaloTag and SNAP-Tag Technologies. ChemBioChem 2020, 21 (14), 1935-1946.

(7) Hori, Y.; Norinobu, T.; Sato, M.; Arita, K.; Shirakawa, M.; Kikuchi, K. Development of Fluorogenic Probes for Quick No-Wash Live-Cell Imaging of Intracellular Proteins. J. Am. Chem. Soc. 2013, 135 (33), 12360-12365.

(8) Plamont, M.-A.; Billon-Denis, E.; Maurin, S.; Gauron, C.; Pimenta, F. M.; Specht, C. G.; Shi, J.; Quérard, J.; Pan, B.; Rossignol, J.; et al. Small Fluorescence-Activating and Absorption-Shifting Tag for Tunable Protein Imaging in Vivo. Proc. Natl. Acad. Sci. 2016, 113 (3), 497-502.

(9) Bruchez, M. P. Dark Dyes-Bright Complexes: Fluorogenic Protein Labeling. Curr. Opin. Chem. Biol. 2015, 27, 18-23.

(10) Gao, J.; Hori, Y.; Shimomura, T.; Bordy, M.; Hasserodt, J.; Kikuchi, K. Development of Fluorogenic Probes for Rapid High-Contrast Imaging of Transient Nuclear Localization of Sirtuin 3. ChemBioChem 2020, 21 (5), 656-662.

(11) Szent-Gyorgyi, C.; Schmidt, B. A.; Creeger, Y.; Fisher, G. W.; Zakel, K. L.; Adler, S.; Fitzpatrick, J. A. J.; Woolford, C. A.; Yan, Q.; Vasilev, K. V.; et al. Fluorogen-Activating Single-Chain Antibodies for Imaging Cell Surface Proteins. Nat. Biotechnol. 2008, 26 (2), 235-240.

(12) Leng, S.; Qiao, Q.; Miao, L.; Deng, W.; Cui, J.; Xu, Z. A Wash-Free SNAP-Tag Fluorogenic Probe Based on the Additive Effects of Quencher Release and Environmental Sensitivity. Chem. Commun. 2017, 53 (48), 6448-6451.

(13) Yu, W. T.; Wu, T. W.; Huang, C. L.; Chen, I. C.; Tan, K. T. Protein Sensing in Living Cells by Molecular RotorBased Fluorescence-Switchable Chemical Probes. Chem. Sci. 2016, 7 (1), 301-307.

(14) Sun, X.; Zhang, A.; Baker, B.; Sun, L.; Howard, A.; Buswell, J.; Maurel, D.; Masharina, A.; Johnsson, K.; Noren, C. J.; et al. Development of SNAP-Tag Fluorogenic Probes for Wash-Free Fluorescence Imaging. ChemBioChem 2011, 12 (14), 2217-2226.

(15) Jung, K. H.; Fares, M.; Grainger, L. S.; Wolstenholme, C. H.; Hou, A.; Liu, Y.; Zhang, X. A SNAP-Tag Fluorogenic Probe Mimicking the Chromophore of the Red Fluorescent Protein Kaede. Org. Biomol. Chem. 2019, 17 (7), 19061915.

(16) Liu, Y.; Miao, K.; Dunham, N. P.; Liu, H.; Fares, M.; Boal, A. K.; Li, X.; Zhang, X. The Cation- $\pi$ Interaction Enables a Halo-Tag Fluorogenic Probe for Fast No-Wash Live Cell Imaging and Gel-Free Protein Quantification. Biochemistry 2017, 56 (11), 1585-1595.

(17) Clark, S. A.; Singh, V.; Vega Mendoza, D.; Margolin, W.; Kool, E. T. Light-Up “Channel Dyes” for HaloalkaneBased Protein Labeling in Vitro and in Bacterial Cells. Bioconjug. Chem. 2016, 27 (12), 2839-2843.

(18) Kang, M.-G.; Lee, H.; Kim, B. H.; Dunbayev, Y.; Seo, J. K.; Lee, C.; Rhee, H.-W. Structure-Guided Synthesis of a Protein-Based Fluorescent Sensor for Alkyl Halides. Chem. Commun. 2017, 53 (66), 9226-9229.

(19) Wang, L.; Tran, M.; D’Este, E.; Roberti, J.; Koch, B.; Xue, L.; Johnsson, K. A General Strategy to Develop Cell Permeable and Fluorogenic Probes for Multicolour Nanoscopy. Nat. Chem. 2020, 12 (2), 165-172.

(20) Grimm, J. B.; English, B. P.; Chen, J.; Slaughter, J. P.; Zhang, Z.; Revyakin, A.; Patel, R.; Macklin, J. J.; Normanno, D.; Singer, R. H.; et al. A General Method to Improve Fluorophores for Live-Cell and Single-Molecule Microscopy. Nat. Methods 2015, 12 (3), 244-250.

(21) Bachollet, S. P. J. T.; Addi, C.; Pietrancosta, N.; Mallet, J.-M.; Dumat, B. Fluorogenic Protein Probes with Red and Near-Infrared Emission for Genetically Targeted Imaging. Chem. Eur. J. 2020, 26 (63), 14467-14473.

(22) Wilhelm, J.; Kühn, S.; Tarnawski, M.; Gotthard, G.; Tünnermann, J.; Tänzer, T.; Karpenko, J.; Mertes, N.; Xue, L.; Uhrig, U.; et al. Kinetic and Structural Characterization of the Self-Labeling Protein Tags HaloTag7, SNAP-Tag, and CLIP-Tag. Biochemistry 2021, 60 (33), 2560-2575. 
(23) Encell, L. P. Development of a Dehalogenase-Based Protein Fusion Tag Capable of Rapid, Selective and Covalent Attachment to Customizable Ligands. Curr. Chem. Genomics 2012, 6 (1), 55-71.

(24) Lukinavičius, G.; Reymond, L.; Umezawa, K.; Sallin, O.; D’Este, E.; Göttfert, F.; Ta, H.; Hell, S. W.; Urano, Y.; Johnsson, K. Fluorogenic Probes for Multicolor Imaging in Living Cells. J. Am. Chem. Soc. 2016, 138 (30), 93659368.

(25) Grimm, J. B.; Muthusamy, A. K.; Liang, Y.; Brown, T. A.; Lemon, W. C.; Patel, R.; Lu, R.; Macklin, J. J.; Keller, P. J.; Ji, N.; et al. A General Method to Fine-Tune Fluorophores for Live-Cell and in Vivo Imaging. Nat. Methods 2017, 14 (10), 987-994.

(26) Frei, M. S.; Tarnawski, M.; Roberti, J.; Koch, B.; Hiblot, J.; Johnsson, K. HaloTag9 : An Engineered Protein Tag to Improve Fluorophore Performance. BioRxiv 2021, doi: 10.1101/2021.04.01.438024

(27) Fares, M.; Li, Y.; Liu, Y.; Miao, K.; Gao, Z.; Zhai, Y.; Zhang, X. A Molecular Rotor-Based Halo-Tag Ligand Enables a Fluorogenic Proteome Stress Sensor to Detect Protein Misfolding in Mildly Stressed Proteome. Bioconjug. Chem. 2018, 29 (1), 215-224.

(28) Hoche, J.; Schulz, A.; Dietrich, L. M.; Humeniuk, A.; Stolte, M.; Schmidt, D.; Brixner, T.; Würthner, F.; Mitric, R. The Origin of the Solvent Dependence of Fluorescence Quantum Yields in Dipolar Merocyanine Dyes. Chem. Sci. 2019, 10 (48), 11013-11022.

(29) Xie, X.; Zuffo, M.; Teulade-Fichou, M. P.; Granzhan, A. Identification of Optimal Fluorescent Probes for GQuadruplex Nucleic Acids through Systematic Exploration of Mono- And Distyryl Dye Libraries. Beilstein J. Org. Chem. 2019, 15, 1872-1889.

(30) Shaya, J.; Collot, M.; Bénailly, F.; Mahmoud, N.; Mély, Y.; Michel, B. Y.; Klymchenko, A. S.; Burger, A. Turn-on Fluorene Push-Pull Probes with High Brightness and Photostability for Visualizing Lipid Order in Biomembranes. ACS Chem. Biol. 2017, 12 (12), 3022-3030.

(31) Péresse, T.; Gautier, A. Next-Generation Fluorogen-Based Reporters and Biosensors for Advanced Bioimaging. Int. J. Mol. Sci. 2019, 20 (24), 6142.

(32) Klymchenko, A. S. Solvatochromic and Fluorogenic Dyes as Environment-Sensitive Probes: Design and Biological Applications. Acc. Chem. Res. 2017, 50 (2), 366-375.

(33) Dumat, B.; Bordeau, G.; Aranda, A. I.; Mahuteau-Betzer, F.; Harfouch, Y. El; Metgé, G.; Charra, F.; FioriniDebuisschert, C.; Teulade-Fichou, M.-P. Vinyl-Triphenylamine Dyes, a New Family of Switchable Fluorescent Probes for Targeted Two-Photon Cellular Imaging: From DNA to Protein Labeling. Org. Biomol. Chem. 2012, 10 (30), 6054.

(34) Iwaki, T.; Torigoe, C.; Noji, M.; Nakanishi, M. Antibodies for Fluorescent Molecular Rotors. Biochemistry 1993, 32 (29), 7589-7592.

(35) Goh, W. L.; Lee, M. Y.; Joseph, T. L.; Quah, S. T.; Brown, C. J.; Verma, C.; Brenner, S.; Ghadessy, F. J.; Teo, Y. N. Molecular Rotors as Conditionally Fluorescent Labels for Rapid Detection of Biomolecular Interactions. J. Am. Chem. Soc. 2014, 136 (17), 6159-6162.

(36) Baranov, M. S.; Solntsev, K. M.; Baleeva, N. S.; Mishin, A. S.; Lukyanov, S. A.; Lukyanov, K. A.; Yampolsky, I. V. Red-Shifted Fluorescent Aminated Derivatives of a Conformationally Locked GFP Chromophore. Chem. Eur. J. 2014, 20 (41), 13234-13241.

(37) Miró-vinyals, C.; Stein, A.; Fischer, S.; Ward, T. R.; Deliz Liang, A. HaloTag Engineering for Enhanced Fluorogenicity and Kinetics with a Styrylpyridium Dye. ChemBioChem 2021, in press, doi: 10.1002/cbic. 202100424. 